\title{
Momentous Crossing of a Solar Boundary
}

\author{
The Parker Solar Probe has entered, for the first time, the Sun's magnetic \\ atmosphere, where it started to gather data that could help researchers \\ solve some of the greatest mysteries of solar physics.
}

\author{
By Christina M. S. Cohen
}

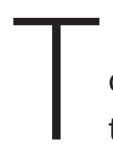

he Universe is full of boundaries that divide markedly different regions. Crossing such boundaries has often led to momentous scientific discoveries. For example, the solar wind was discovered when Soviet spacecraft left Earth's magnetosphere and entered the interplanetary medium. Those missions showed that the plasma flowing from the Sun was a fast-paced and highly variable "wind" rather than a slowly expanding "breeze." The crossings of the heliopause by NASA's

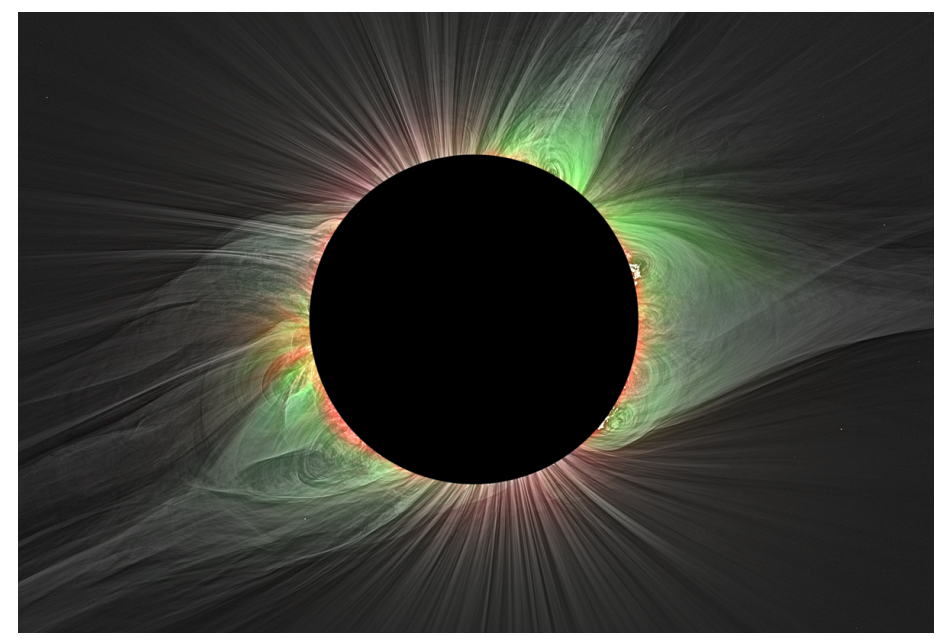

Figure 1: Image of the solar corona during a total solar eclipse on Monday, August 21, 2017, above Mitchell, Oregon. (The red light is emitted by charged iron particles at 1 million degrees Celsius and the green are those at 2 million degrees Celsius.) On April 28, 2021, NASA's Parker Solar Probe crossed the so-called Alfvén surface, entering, for the first time, a part of the solar corona that is "magnetically dominated."

Credit: S. R. Habbal et al., Astrophys. J. Lett. 911, L4 (2021)
Voyagers were similarly momentous, taking the spacecraft from the heliosphere, a region dominated by the solar wind, to interstellar space. Voyager observations revolutionized our understanding of the heliosphere's interaction with interstellar space. Today, NASA reports on the accomplishment of another historic crossing: In April 2021, the Parker Solar Probe (PSP) flew closer to the Sun than any previous mission, becoming the first spacecraft to enter a part of the Sun's corona whose behavior is dominated by magnetic fields [1] (Fig. 1). In the coming few years, increasingly tighter orbits will bring PSP even closer to the star, providing more opportunities to explore the magnetically dominated corona and thereby answer some of the biggest questions in solar physics.

The boundary crossed by PSP is called the Alfvén surface-defined as the point where magnetic and kinetic energies balance. Above this surface, the solar wind streams freely away from the Sun. Below the surface, magnetic fields dominate the motion of the plasma, binding it to the Sun. The two regions can be distinguished by the solar wind speed, which, at the boundary, is equal to the Alfvén speed (the speed at which hydrodynamic waves travel in a magnetized plasma). The wind blows slower (sub-Alfvénic) below the surface and becomes faster (super-Alfvénic) above. The transition between the two regions involves important changes in the solar wind. In particular, the plasma waves above the Alfvén surface cannot travel fast enough against the outward moving solar wind to reach back to the solar corona, effectively "disconnecting" the outgoing solar wind and the magnetically dominated corona [2].

Today, we routinely observe the plasma and fields of the solar 
wind in situ with a fleet of spacecraft located far from the magnetically dominated corona. We also observe the Sun's atmospheric layers-from the photosphere to the chromosphere to the corona-via remote imaging techniques performed at various wavelengths by both space- and ground-based instruments. These combined observations have allowed researchers to identify various types of solar wind (slow and fast) and various types of solar wind phenomena, such as magnetic clouds, and to correlate them with features in the solar atmosphere, such as coronal holes and solar flares.

While we can infer the location of the Alfvén surface from these data, little is known about the processes that occur at and below the surface. Researchers suspect that the region may hold the answers to many open questions, including how the corona is heated, how the slow solar wind is generated, what causes solar wind ions to be heated more effectively than electrons, and what produces magnetic switchbacks-rapid reversals in the magnetic-field direction observed by PSP [3] and previous missions. Up to now, all of our observations have been made far from the actual boundary, but the information needed to solve these puzzles can only be gathered by crossing the Alfvén surface and exploring this region in situ.

Scientists have thought about sending a spacecraft to "touch the Sun" since the late 1950s. Getting there isn't the main obstacle: Suitable orbits can be achieved using "gravity assists" from Solar System planets, a trick exploited since the first interplanetary space missions. Such assists will be provided by seven Venus flybys over the course of 24 orbits, reducing PSP's perihelion (distance of closest approach to the Sun) from 35.7 solar radii $\left(R_{\odot}\right)$ to a final $8.9 R_{\odot}$ (expected in 2024), well within the Alfvén surface. The real challenge, instead, is surviving the journey, which requires technology that only recently became available (see How to Survive Flying Too Close to the Sun). At $10 R_{\odot}$ the spacecraft will be subject to a flux of light, or irradiance, that's 475 times larger than the solar irradiance at Earth. PSP uses a specially designed heat shield to create a protective shade, with the spacecraft automatically reorienting itself to keep its instruments and body within the shield's shade. Like Earth's magnetosphere and the heliosphere, the Alfvén surface is not a static, spherical surface, as the corona undergoes large, continuous changes in plasma density and magnetic-field configuration. Researchers had tried to anticipate when and where PSP might encounter the Alfvén

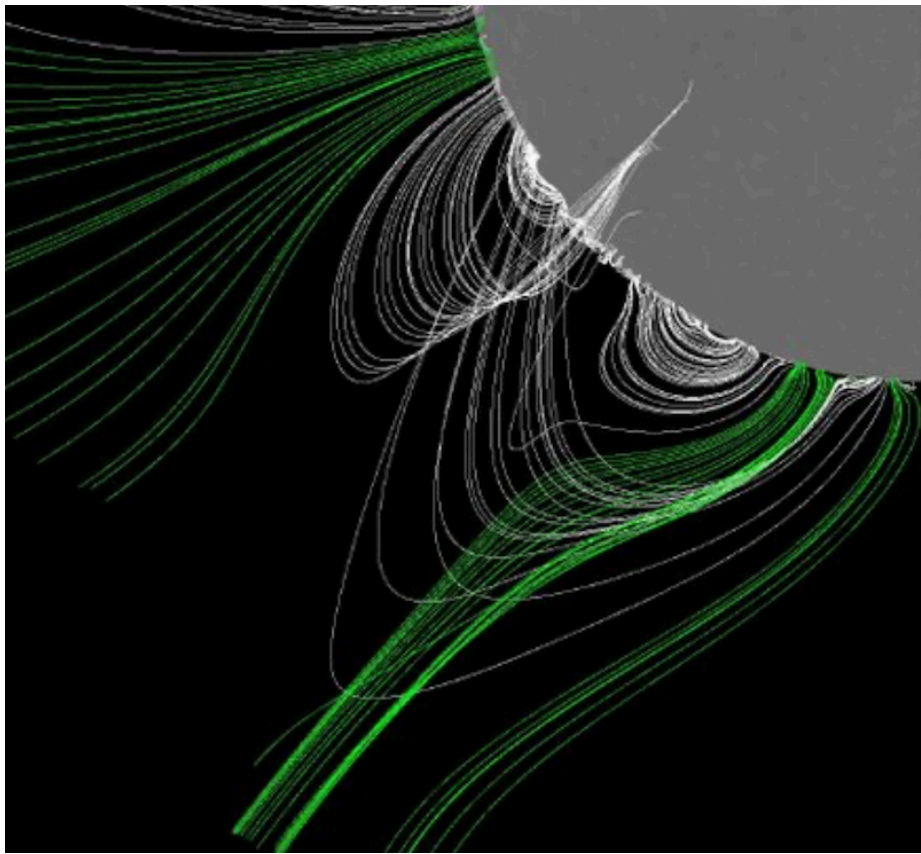

Figure 2: Model representation of a pseudostreamer, in which magnetic-field structures (in green) stream out of regions with the same field polarity, separated by an inner region with opposite polarity, with the different-polarity regions connected by closed field lines (white). A pseudostreamer can move up the location of the Alfvén surface, explaining why PSP encountered the surface at a distance greater than the spacecraft's perihelion.

Credit: O. Panasenco et al., Sol. Phys. 391, 287 (2012)

boundary. The most recent prediction-a boundary crossing around $20 R_{\odot}$ in late 2020 -turned out to be inaccurate, but it wasn't far off. On April 28, 2021, during its eighth orbit, PSP crossed the Alfvén surface around $18 R_{\odot}$ and flew through the magnetically dominated solar corona.

Justin Kasper from BWX Technology and the University of Michigan and colleagues observed three intervals in which PSP measured sub-Alfvénic solar wind velocity and low plasma densities-suggestive of the boundary crossing. The last two of these periods were short, but the first, which lasted nearly 5 hours, was exceptional: The observations that the thermal and kinetic energy of the particles was dominated by the magnetic energy and that the magnetic pressure was high provided unambiguous evidence that PSP had indeed crossed into the magnetically dominated solar corona. 
When PSP crossed the Alfvén surface, it was not at its perihelion. To explain why the crossing occurred at this location, the authors modeled the magnetic structures of the corona. The analysis indicated that PSP passed through a pseudostreamer [4] (Fig. 2). Streamers and pseudostreamers are large-scale magnetic structures in the Sun's corona.

Pseudostreamers are formed across nearby areas with the same magnetic polarity separated by a patch of opposite polarity. The center of a pseudostreamer is a region of enhanced magnetic field and reduced plasma density. This combination can push the Alfvén surface higher up in the corona, explaining why PSP's orbit was able to cut across it.

The period of time PSP spent below the Alfvén surface was too short to fully characterize the boundary and explore the inner region. Researchers expect that such a full characterization will require multiple expeditions carried out over different magnetic configurations and solar conditions. But there will be several future opportunities. The last perihelion, reached in November 2021, was at a mere $13.6 R_{\odot}$. At such a height, PSP likely crossed the Alfvén surface again and perhaps spent much more than 5 hours in the magnetically dominated solar corona.

However, we don't yet know what happened during this last close approach. Full communication can only occur when PSP is far enough from the Sun for it to be safe to turn and point its high-gain antenna toward Earth. That data transfer is expected in late December of this year. PSP researchers are surely looking forward to these data as a highly anticipated holiday gift. Meanwhile, the entire scientific community awaits the discoveries that may result from finally touching the Sun.

Correction (26 December 2021): The Fig. 1 caption was corrected to indicate that the image was taken in Mitchell, Oregon. The figure credit was also corrected.

Christina M. S. Cohen: Space Radiation Lab (SRL), California Institute of Technology, Pasadena, CA, USA

\section{REFERENCES}

1. J. C. Kasper et al., "Parker Solar Probe enters the magnetically dominated solar corona," Phys. Rev. Lett. 127, 255101 (2021).

2. C. E. DeForest et al., "Inbound waves in the solar corona: a direct indicator of Alfvén surface location," Astrophys. J. 787, 124 (2014).

3. S. D. Bale et al., "Highly structured slow solar wind emerging from an equatorial coronal hole," Nature 576, 237 (2019).

4. Y.-M. Wang et al., "Coronal Pseudostreamers," Astrophys. J. 658,1340 (2007). 